\title{
Editorial
}

\section{Novel Topics in Inflammatory Bowel Disease}

\author{
Atsushi Sakuraba, ${ }^{1}$ Alessandro Armuzzi, ${ }^{2}$ Kian Keyashian, ${ }^{3}$ and Makoto Naganuma ${ }^{4}$ \\ ${ }^{1}$ Section of Gastroenterology, Hepatology and Nutrition, University of Chicago, 5841 S. Maryland Avenue, \\ MC 4076, Chicago, IL 60637, USA \\ ${ }^{2}$ IBD Unit, Complesso Integrato Columbus, Gemelli Hospital-Catholic University Foundation, 00168 Rome, Italy \\ ${ }^{3}$ Division of Gastroenterology, Oregon Health \& Science University, Portland, OR 97239, USA \\ ${ }^{4}$ Department of Gastroenterology and Hepatology, Keio University School of Medicine, Tokyo 160-8582, Japan \\ Correspondence should be addressed to Atsushi Sakuraba; asakurab@medicine.bsd.uchicago.edu \\ Received 11 January 2016; Accepted 11 February 2016
}

Copyright (c) 2016 Atsushi Sakuraba et al. This is an open access article distributed under the Creative Commons Attribution License, which permits unrestricted use, distribution, and reproduction in any medium, provided the original work is properly cited.

We are pleased to announce the publication of the special issue focusing on novel topics in inflammatory bowel disease. It gained the interest of researchers from all over the world and more than 20 articles were submitted for review. Among them, our editorial team consisting of four renowned researchers in this field has selected six articles for publication. This includes basic and clinical research studies as well as translational research focusing on mechanism, epidemiology, treatment outcomes, and genetics. We are confident that this special issue advances the understanding and research of inflammatory bowel disease.

\section{Acknowledgment}

We would also like to thank all the reviewers who have participated in the review process of the articles submitted to this special issue.

Atsushi Sakuraba Alessandro Armuzzi

Kian Keyashian Makoto Naganuma 


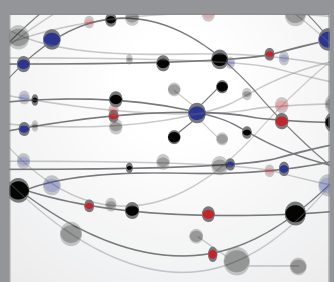

The Scientific World Journal
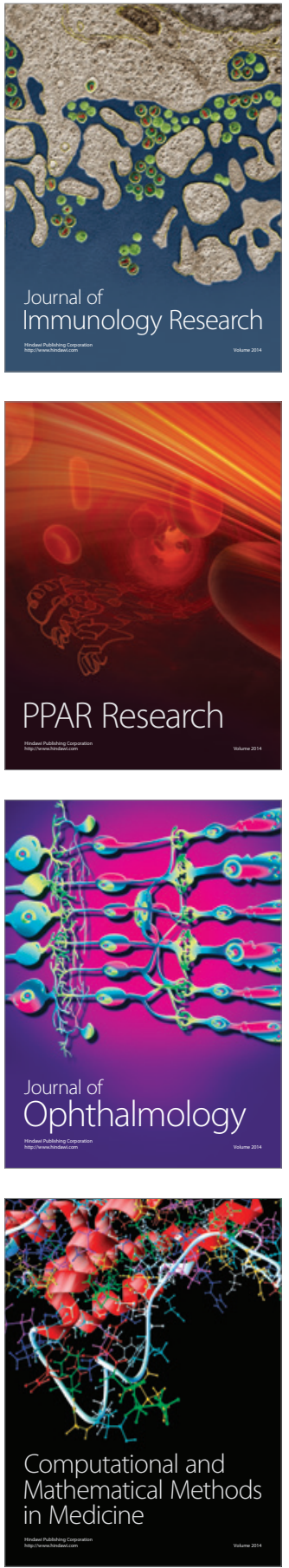

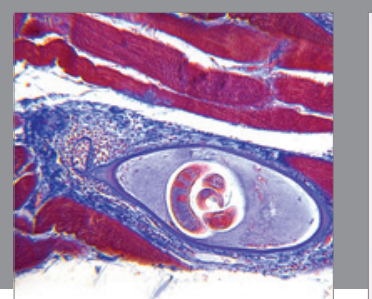

Gastroenterology Research and Practice

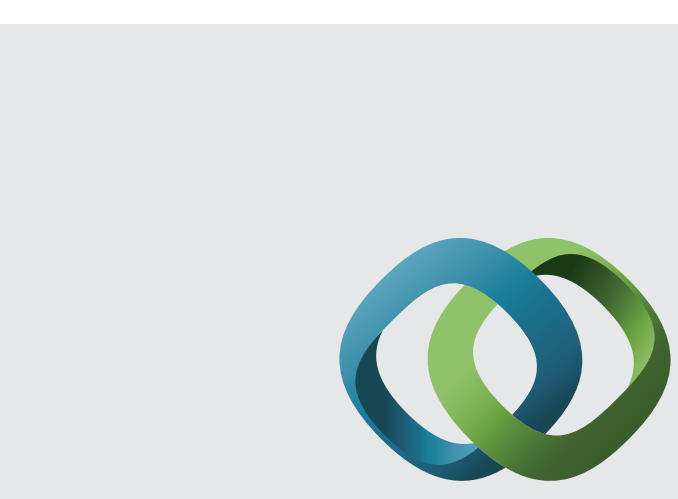

\section{Hindawi}

Submit your manuscripts at

http://www.hindawi.com
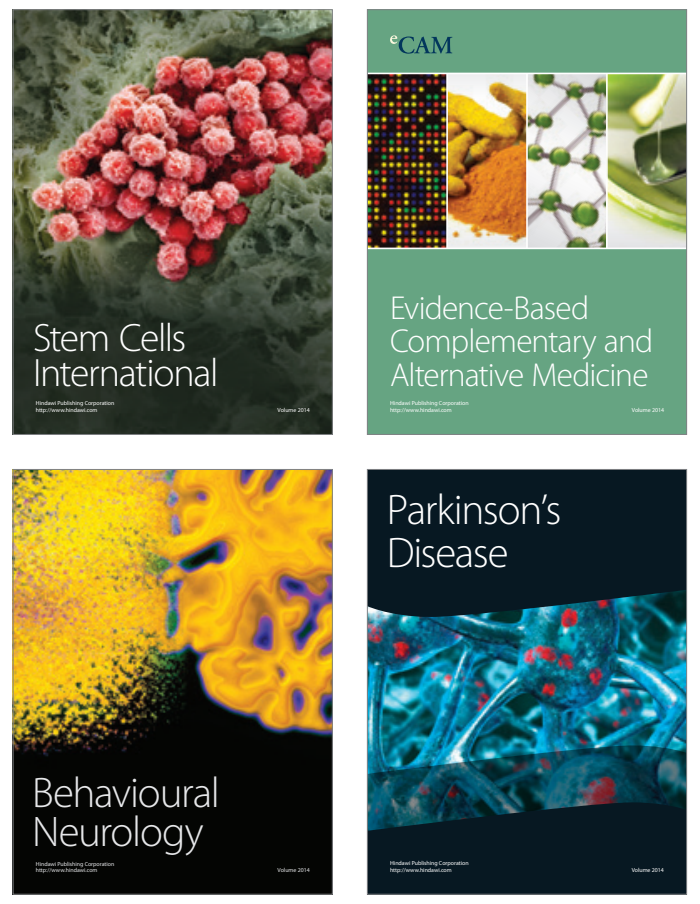
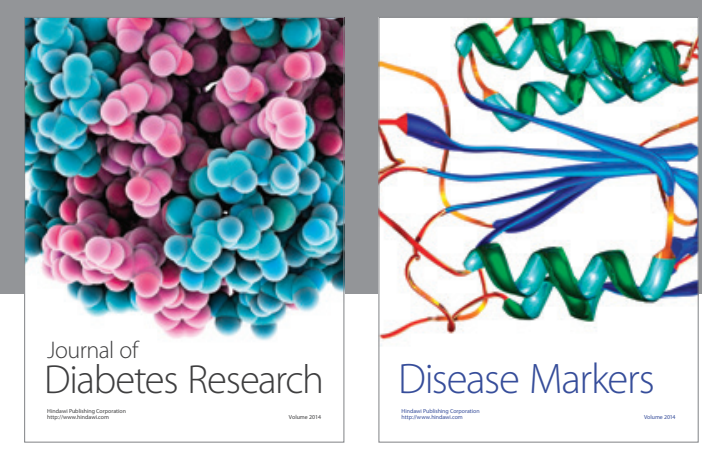

Disease Markers
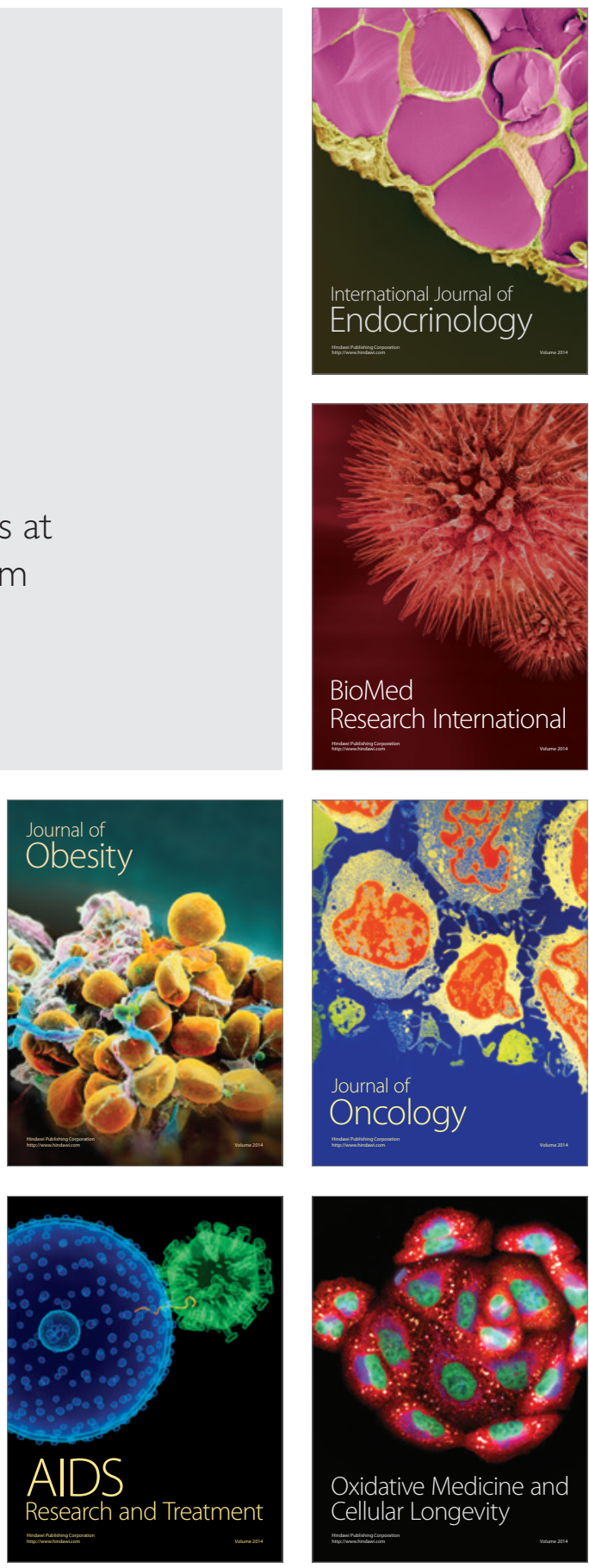